\title{
Selling the Past and the Present Alike: Streaming Ballet for Live Audiences during Lockdown
}

\author{
Arianna Maiorani*(Loughborough University)
}

\begin{abstract}
During the Covid-19-induced lockdown, most of the main theatres and artistic companies in Europe and North America kept on proposing ballet seasons to homebound audiences who could connect to online channels and enjoy 'live' a performance in streaming. At a certain point, though, what was actually streamed was not a live performance but a recording that would have been otherwise available on DVD. What type of live performance illusion was therefore proposed to these theatre audiences all over the world? Did this phenomenon mark the beginning of a new way of defining, framing and understanding a live theatre performance? These are some of the questions that this paper intends to propose about the fruition of theatre performances, and in particular of dance ones, in the pandemic era and perhaps beyond.
\end{abstract}

Key words: Covid-19, Ballet, Online, Streaming, Iive Performance

\section{Introduction}

When Covid-19 induced lockdown started in England and in the rest of Europe in March 2020, for theatres and ballet, opera, and drama companies all over the continent and beyond it was like going back to 'the plague times'. Live entertainment stopped all at once and many smaller or more fragile artistic groups did not manage to re-emerge from the first long period of inactivity. Many bigger entities, however, found a way to reinvent their lost seasons and consolidate their audience affection and support through several types of engaging events that ran not only via on demand video channels but also through social media live streaming. Some ballet companies and musicians, for example, shared their lockdowns

\footnotetext{
* Loughborough University, Professor, A.Maiorani@lboro.ac.uk
}

with communities of fans and like-minded practitioners or amateurs by providing free access to Facebook streaming sessions of ballet classes, music master classes, yoga, physical and mental training, all delivered from the artists' and professionals' own homes.

This phenomenon spread all over the world, much more than the cinema live streaming seasons that had become quite popular before the pandemic. It is a phenomenon that caused a reconsideration of the concept of live performance, already problematised and discussed in relation to the development of technology and new media well before 2020. It is a phenomenon that invites a serious reflection on the factors that define a live performance for an audience, factors that involve a shared perception of time and space and their configurations (or re-configurations), 
factors that determine the creation of new types of experiences and that impact on the traditional and still ongoing debate about the difference between a live performance and its document in the form of a recording.

This paper will address this phenomenon in its different aspects specifically in relation to ballet performances and with examples set by European and North-American companies of international reputation.

\section{A three-phase project of reinvention}

Studies on the concept of live performance and on its relationship with the evolution of audience configurations and technology predate the pandemic and show that the lockdown was simply the triggering event that perhaps accelerated a transformation that was ready to happen. In 2012, reflecting on his own previous work, where he started by reflecting on the difference between live and mediatized performance, ${ }^{1)}$ Philip Auslander defined the period through which the evolution of the term 'live' has occurred:

"the idea of what counts culturally as live experience changes over time in relation to technological change (..) liveness is not an ontologically defined condition but a historically variable effect of mediatization. It

1) "I often employ the term "mediatized", admittedly somewhat loosely, to indicate that a particular cultural object is a product of the mass media or the media technology. "Mediatized performance" is performance that is circulated on television, as audio or video recordings, and in other forms based in technology of reproduction." (Auslander, 1999: 5). was the development of recording technologies that made it both possible and necessary to perceive existing representations as "live." Prior to the advent of these technologies (e.g., sound recording and motion pictures), there was no need for a category of "live" performance, for that category has meaning only in relation to an opposing possibility. The history of live performance is thus bound up with the history of recording media, and extends over no more than the past 100 to 150 years." (Auslander, 2012: 3).

Mediatized and not mediated, as the focus is on the process through which the performance is conveyed to the audience through a medium, not on the workings of the medium itself. ${ }^{2)}$ Auslander, however, is not happy to simply define the concept of liveness in relation to the mediatization of performances and recognises the complexity of such a notion: the difference is not only the result of the employment of a broadcast medium; the change from live performance to mediatized also encompasses, for example, the difference between a live broadcast and the broadcast of a performance that is recorded live 3 )

2) In relation to mediation and technology, Susan Davis writes: "Mediation therefore involves subjects (or participants) working towards achieving certain goals, objects and outcomes, through mediated action involving signs, tools and artefacts. What this means is that the object or idea of what a subject wants to achieve may be held internally within their mind, however, realisation of these ideas and goals relies upon external mediation and interactions with various tools. Tools include culturally learned processes or conceptual tools, signs such as language, as well as physical tools and technologies." (Davis, 2012: 504).

3) "Another use of the term worth considering is in the phrase recorded live. This expression comes even closer to being an oxymoron (how can something be both recorded and live?) but is another concept we now accept without question. In the case of live recordings, the audience shares neither a temporal frame nor a physical location with the performers, but experiences the performance later and in a 
and cannot be based on the traditional recognition that a live event, in order to be considered as such, needs to involve the co-presence in space and time of performers and audience. In this perspective, 'live' is not a concept that can be restricted to temporal and spatial coordinates: it is rather a quality of experience.

Auslander's work focuses on all types of performances, artistic, sports, digital, even the perception of websites becoming live, which shows the enormous cultural importance of this concept and its fundamental role in the advertising and marketing strategies that accompany the commercialisation of these events.

Live broadcasts of theatre, opera and ballet productions have become more and more popular since the Metropolitan Theatre in New York started streaming its Opera season through selected cinemas across the world in 2006. Its example was soon followed by other opera houses in the world and international theatres and ballet companies.4) The

different place than it first occurred. The liveness of the experience of listening to or watching the recording is primarily affective: live recordings allow the listener a sense of participating in a specific performance and a vicarious relationship to the audience for that performance not accessible through studio productions." (Auslander, 2012: 5).

4) Some more data about the wave of theatre broadcast that followed the MET live broadcast season in 2006: "On 30 December 2006, the New York Metropolitan Opera transmitted a live performance of The Magic Flute into 88 cinemas in North America and 10 overseas ( $\cdots)$ The 6 transmissions of the first season grew quickly to 10 , and by 2012-2013 were being shown live in nearly 2000 cinemas in 64 countries, with an attendance of over 2.5 million $(\cdots)$ The Met was followed into cinemas on a regular basis by the season opening performance at La Scala, Milan, and by performances in England from the Glyndebourne Festival, the Royal Opera, and the English National Opera, in Spain from the Liceu, Barcelona, and the Teatro Real, Madrid, and from Opera Australia $(\cdots)$ There have been regular live transmissions of ballet performances by the Bolshoi Ballet, the Paris Opera Ballet and the Royal Ballet and from the Mariinsky Theatre in Leningrad (*) By 2013-2014, NTL
National Theatre in London was particularly successful, as well as the Bolshoi Theatre, with live broadcasts of famous ballet productions; the Royal Opera House with Opera and ballet; the English National Ballet with ballet premieres of new choreographies. These are just a few examples of what I would define as the first phase of live performance transformation into a different type of event.

As also observed by Auslander (2012), in the case of live broadcasts, the performers and the audience may not share the same space in terms of performance environment anymore but they do share the same time as the audience watches the show at the same time it is performed. Moreover, rather than trying to make the live broadcasts fit the new and different environments of cinema theatres, the opposite happened and cinema goers were offered even more insights and 'glimpses' behind the scenes than a traditional audience on location would be able to enjoy.

Live broadcasts are usually conducted by a presenter who introduces the theatre and its history as well as the performance and also manages to show bits of pre-show rehearsals and carry out live interviews with performers and relevant artists. It is almost as if the cinema audience was privileged. These 'extras' offered to them also mark a difference between two audience spaces and, consequently, two audience experiences of the same live representation: the on location audience, whose experience is limited by the traditional boundaries between stage space and

had grown to eight performances, shown in 35 countries to an audience of 1.49 million, about $40 \%$ of whom were outside the UK ( $\cdots)$ In 2013, the Royal Shakespeare Company began live transmissions of several productions a year to cinemas. (King, 2018: 220-221). 
audience space and, quite literally, by the view allowed by the physical seating plan; and the live streaming audience, who pay less, attend the performance in a less prestigious environment but can cross those traditional space boundaries via the interpolation of a medium, which also grants a view of the performance that is the same for everybody (the eyes and perspective of the camera and the camera operators) but in many ways better (closer, neater) than what is available to most of the audience on location. The audience on location experience is diversified by different ticket prices and how much each person can afford. The live broadcast audience is homogenised by the camera lenses. The two audiences do occupy different spaces, which determine different experiences of the same live performances.

The clever distribution through cinema chains and the comparatively cheaper price of the tickets brought these live broadcast seasons such a success that theatres and companies had to start offering 'replica' shows: live recordings of the streaming sessions were offered for the audience who did not manage to attend the live broadcast in the same cinemas, and at a slightly lower price. These replicas mark the start of the second phase of the live performance transformation: a move towards replica streaming only for the cinema audience who does not share the space nor the time of the show which was recorded (and once broadcast) live. What quality of the 'live' event is being sold and experienced at this point?

The offer of shows recorded live precedes the live streaming seasons in cinemas. Live recorded drama has been offered on television for decades in Europe, with particular peaks of success in Italy, England, and France in the 1960s and 1970s. The 'live' quality of these broadcasts lies also in the 'thrill' offered to home audiences by the uncertainty: nothing can (or should) be edited and the 'live focus' is on the 'replicated live time' rather than on the space sharing. This type of experience requires more effort from the audience as they need to 'forget' that they are watching a recording; this is however a condition that is easier to forget when one has to go 'live' to the cinema to watch these replicas, which enhances the live quality of the experience. It also offers the possibility of watching a quality theatre production to a wider audience (in many cases to entire families) who cannot afford to go to a specific theatre for logistic and/or economic reasons.

The 2020 pandemic triggered what I would define as the third phase of the live performance transformation: the 'live at home'. In the UK, when live performances were forbidden for Covid-19 restrictions, a series of events were organised by prestigious companies like the Royal Opera House and the Royal Ballet as 'live streaming' sessions, which were actually one-off broadcasts of performances recorded live and then edited in post-production, usually available in DVD as live. Some of these events were broadcast for free, mostly at the beginning as 'teasers', others were charged. This situation differed from that where the audience went to the cinema: these streaming events were created for home viewing in the attempt of keeping the experience of attending a theatre performance alive as well as providing some continuity in the contact with the audience. It was also a way for generating income when no real live performance was permitted.

However, the true third-phase transformation occurred on Facebook and Twitter where, at the same 
time as the 'home' broadcasts, companies like the English National Ballet started streaming classes from dancers' and masters' homes on these social media platforms, all for free and varying from classical dance classes to yoga and pilates. The same was done by some musicians who even broadcast live master classes on concert performance (one excellent example is pianist Boris Giltburg, who also performed for free through the same channels). These classes ran almost daily and were nothing like the somewhat staged and edited broadcasts that are run throughout the world during yearly events like the World Ballet Day. These regular broadcasts created a situation where performers were performing what is not usually a performance but rather a service (the delivery of a class) from their private home spaces to audiences in their own home spaces: performers and audiences did not share the same physical space but they did share the same type of space and the same personal as well as socio-historical experience of it. They also shared the same time. This combination created a situation of live pandemic experience as a new type of artistic and interactive live event as many people did not watch the classes to actually take them but just out of interest.

A side effect of the creation of this type of experience was the emergence of a new type of audience who enjoyed following live broadcast events when performances were permitted once more but under severe restrictions, which allowed only for reduced audiences, like the "Emerging Dancer" award soirée organised by the English National Ballet at the end of September 2020 and accessible via streaming through a very affordable ticket on several digital platforms.

In the case of one famous pre-pandemic performance event, however, what I defined as the first, second and third phases of live performance transformation converged in what I would describe as a mediatic franchise event. I am talking about the 1992 worldwide live broadcast of Tosca: in the Settings and Times of Tosca (Tosca: nei luoghi e nelle ore di Tosca), a live performance of Giacomo Puccini's opera that was performed, broadcast 5 ) and recorded live in the locations where the story actually takes place and at the times of the day when the events actually unravel in the plot. This event was a true reification of one of the masterpieces of the great Italian maestro: directed by the eminent theatre director Giuseppe Patroni Griffi and with the cinematography of Academy Awards winner Vittorio Storaro, the cast included Placido Domingo (Mario Cavaradossi), Catherine Malfitano (Floria Tosca) and Ruggero Raimondi (Baron Scarpia) accompanied by the Orchestra and Chorus of RAI (Radio Televisione Italiana) conducted live by Zubin Metha in a separate location. Whereas this live performance did not allow for any genuine on location audience, millions of people worldwide watched the live broadcast that took place over the course of a whole day (the first act in the morning, the second act in the evening and the third act at dawn) sharing the time of the show as well as that of the plot in a unique live temporal experience.6) The whole opera was later on broadcast as a live recording on television and in 1993 the live recording was sold in DVD format with some post-production editing (for example, the famous fall occurred to Placido Domingo in the first act, one big

\footnotetext{
5) The broadcast covered 107 countries in five continents.

6) This would be a good example of what Scott McQuire and Natalia Radywyl call "the increasing implication of art in complex temporalities”. (McQuire and Radywyl, 2010: 6).
} 
'live thrill', was cut). This exceptional example shows even more that it is the audience perception and experience of live time or space that actually determines the 'live quality' of a performance and that the distinction between a live performance and its document (a video and/or audio recording) cannot be simply based on the sharing of spatio-temporal coordinates but needs to be recognised as a much more complex relationship between the spatiotemporal coordinates of the performance and those of the audience's experience.

Tosca: in the Settings and Times of Tosca obviously inspired all the projects in the same line that followed. A much more recent example, another pre-pandemic one, is the 2013 production of Shakespeare's A Midsummer Night's Dream by the Royal Shakespeare Company. The play was performed in real time in front of an on location audience during three days by the company and the finale coincided with the actual Summer solstice.7) The whole performance was also streamed live online: another example where two types of audiences are differentiated not by the shared time, which was the same, but by the shared space, which was home for those who attended the event online. What made this event different, however, was the creation of a new group of characters played simultaneously by actors who shared their stories online, thus creating a parallel event for online audiences that expanded the world and reach of the original Shakesperean play. The new content was created and shared on the Google + platform. In this way, the event franchise created by Tosca: in the Settings and Times of Tosca was taken to another

7) See Lonergan 2016 for more information. level, which perhaps could be compared to the blockbuster film franchises that spawn not only a very rich market of by-products in the form of gadgets but also spin-off films and tv series. The pandemic restrictions and lockdowns throughout the world only triggered further developments of this type of broadcasts and the creation of a wider and more complex audience. The project was called A Midsummer Night's Dreaming, where the continuous tense of the verb 'to dream' evokes not only the duration and live quality of the performance itself but also the continuity of its effect and impact on the audience and the users who kept on generating content on the Google + platform. Can we now talk of this phenomenon as the creation of a new artistic experience franchise?

\section{The particular case of dance performances}

In 2014 Claire Read was already observing the effects on theatre audiences of the growing phenomenon of live streamed performances:

"Live streaming enables performances to be accessed by viewers in discrete locations, offering a sense of liveness despite the inevitable distance between the performers and the digital audiences. "(Read, 2014: 68)

Read's article focuses on drama performances but it is relevant to a reflection on all types of theatre performances as it mentions also Auslander's reconsiderations of the use and understanding of the term 'live', ${ }^{8)}$ which has repercussions not only in the

8) "Because liveness has been disrupted by mediatization, such 
creation, marketing and experience of different broadcast theatre events but also in the documentation and archival of such events. In fact, whereas in the past the recorded document of a performance was considered as everything but a 'live' version of it and was for the same reason considered as almost something 'other' than it, with the streaming of live-recorded performances that is advertised and experienced as a 'live' event, it is not only the relationship between audience and the sense of a live performance that changes but also the criteria that traditionally defined the archived recording of a performance and that now define instead something that is being broadcast and experienced as 'live'.

Thus, theatre streaming events become franchises of performances that go 'live' for a certain period (i.e. they are accessible via digital streaming for a specific period of time) and that in this way acquire those spatio-temporal finite coordinates that characterised in the past only the understanding and experience of live, on stage performances. Theatre has thus expanded into a cycle of transformation and renewal that seems to have upgraded its features to the digital era.

It must however be recognised that not all types of performances are impacted in the same way by the home streaming transformation. What is the impact of this type of streaming on the reception of ballet choreography? How does the visualisation of

as with the example of live streams that disturb the spatial closeness between the performers and audience, a confusion concerning the definition of liveness has evolved as events that offered co-temporal and co-spatial liveness were labelled as live, in addition to performances that could not offer both co-temporal and co-spatial liveness but offered instead one element of liveness or an overall 'sense' of liveness." (Read, 2014: 68) close-ups and cornered views affect the audience that was used to the view of an entire stage where the distance and involvement separating dancers and viewers was not mediated by the spoken word but determined by the way the dancers' bodies projected in the performance space?

Projection is a term that I coined to explain in a systematic way how dancers create meaning for their audiences by interacting with the performance space through choreographed movement. As an interactive connection that generates movement-based communication,9) projection happens when the dancers' body parts project towards meaningful portions of the performance space and it is at the basis of my Functional Grammar of Dance, an analytical tool for capturing dance discourse through the systematic analysis of choreographed motivatedmovement structures. Dance choreographies engage the audience on the basis of this interactive, dynamic principle to narrate, describe, express feelings, thus creating a connection between the audience in a theatre and the performers as well as the performance space. With streamed ballet, the space of this interaction is embedded in the home space and experienced as 'live' as it has acquired spatio-temporal coordinates, not through the physical sharing of the theatre environment. Sometimes, close-ups make it difficult to understand where or to which bodies or objects dancers are projecting as the camera eye tends to misunderstand a ballet performance for a drama or a film and forgets that dancers do not express themselves through words or mainly through facial expressions. The fact that dance means through

9) See Maiorani 2020 for the full account and application of the Functional Grammar of Dance and the use of the notion of projection in Kinesemiotics. 
projections, through the physical interaction of the human body with the performance space that is shared as such in the performance environment of a theatre, impacts in a very substantial way on the experience of dance performances as live streaming events. Perhaps, in dance performances more than in every other type of performance, the process of mediatization occurring through the streaming is so unavoidably evident that the experience can never be that 'live'.10) It can allow insights that are not possible in a theatre but diminishes the force and immediacy that is experienced by the audience through projections: which problematises even more the notion of 'live' that is now being used for the streaming seasons of many theatres and companies worldwide during the pandemic.

\section{The new 'live'? Concluding} remarks on re-presentations

\section{of representations}

Space and time as represented by theatre performances are re-presented at home but with an eye that does not take int consideration the actual physical perspective of a theatre audience. The

10) In his early work, Auslander also suggests a case-by-case reconsideration of the difference between live and mediatized performances: "In challenging the traditional opposition of the live and the mediatized, I am not suggesting that we cannot make phenomenological distinctions between the respective experiences of live and mediatized representations, distinctions concerning their respective positions within cultural economy, and ideological distinctions among performed representations in all media. What I am suggesting is that any distinctions need to derive from careful consideration of how the relationship between the live and the mediatized is articulated in particular cases, not from a set of assumptions that constructs the relation between live and mediatized representations a priori as a relation of essential opposition." (Auslander, 1999: 54). transformation of theatre live broadcasts into events that are more similar to films, including the implications this might have on audience reconfigurations, has also attracted the attention of marketing studies ${ }^{11)}$ in relation to competition and the creation of new markets as well as of new challenges for those artistic entities that are too small to be able to afford performance broadcasts. Even though the camera might work in many cases in more or less the same way, the difference between a film and a live broadcast of a theatre performance mostly lies in the immediacy and uncertainty and lack of editing that characterise the performance. That is where the 'thrill' of liveness lies and that is the quality that live streaming sessions try to reproduce by providing spatio-temporal coordinates to non-live events, coordinates that can still evoke that immediacy. These new theatre 'liveness' is achieved through a process that could be defined as the re-presentation of the representation, which unveils a different meta-dimension of the theatre. It is the meta-liveness that characterises the theatre streaming seasons in the pandemic era and that will perhaps become a new, permanent feature of the way audiences will experience theatre even beyond this particular historical period.

11) “An emphasis on 'delivery' suggests that the main attraction of the cinema version for its audience is to make a particular performance, or at least a particular art form, available at lower cost in time and money than its stage parent. If so, the principal market impact of streaming would be on demand for live performances of that art-form $(\cdots)$ the transmitted version provides a significantly different aesthetic experience from its live performance on stage ( $\cdots)$ it shares important aesthetic characteristics with all cinema films, irrespective of subject matter. This suggests the possibility that the main competitors for the time and money of potential audiences may not be the parent stage performance, or stage performances at all, but other events in a cinema." (King, 2018: 222). 


\section{References}

Auslander, Philip. 1999. Liveness. London-New York:

Routledge.

Auslander, Philip. 2012. "Digital Liveness: A Historico-Philosophical Perspective.” PAJ: A Journal of Performance and Art, 34(3, September), 3-11.

Davis, Susan. 2012. "Liveness, mediation and immediacy - innovative technology use in process and performance." Research in Drama Education: The Journal of Applied Theatre and Performance, 17(4), 501-516.

King, Timothy. 2018. "Streaming from stage to screen: its place in the cultural marketplace and the implication for UK arts policy." International Journal of Cultural Policy, 24(2), 220-235.

Lonergan, Patrick. 2016. Theatre \& Social Media. London: Palgrave.

Maiorani, Arianna. Kinesemiotics: Modelling How Choreographed Movement Means In Space. London-New York: Routledge.

McQuire, Scott and Natalia Radywyl. 2010. "From Object to Platform Art, digital technology and time." Time and Society, 19(1), 5-27.

Read, Claire. 2014. “'Live, or almost live...': the politics of performance and documentation." International Journal of Performance Arts and Digital Media, 10(1), 67-76. 
